\title{
High Capacity Watermarking Using Balanced Multiwavelet Transforms
}

\author{
Lahouari Ghouti, Ahmed Bouridane, and Said Boussakta
}

\begin{abstract}
The emergence of digital multimedia and the proliferation of its use have raised major concerns about the protection of intellectual property. In response to these concerns, digital watermarks have emerged as a possible solution for protecting the intellectual property of digital content. In this paper, we derive estimates of data-hiding capacity of balanced multiwavelet transforms. This class of transforms, relatively new, has useful properties for image processing applications as shown in this paper. Furthermore, we will investigate the relevance of two closely related statistical models, developed for scalar wavelets, for modeling the statistics of balanced multiwavelet transform coefficients. Finally, we will present performance results of a spread spectrum watermarking system that is based on this new transform.
\end{abstract}

\section{INTRODUCTION}

With the rapid growth and widespread use of network distributions of digital media content, there is an urgent need for protecting the copyright of digital content against piracy and malicious manipulation. Recently, watermarking systems have been proposed as a possible and efficient answer to these concerns. Digital watermarking refers to embedding an auxiliary signal (called watermark) within a host signal such as text, audio, image, or video. Tampering includes operations such as lowpass/highpass/nonlinear filtering, compression, addition of noise, resampling, format conversion, and desynchronization. Tampering operations could be accidental due to lossy compression or transmission over noisy communication channel as well as intentional due to an attacker. In the case of intentional tampering, the attacker applies a variety of data processing operations in order to severely limit the ability of the decoder to recover the embedded watermark signal. Information hiding methods have numerous applications such as covert communications, authentication, proof of ownership, customer tracing, and data embedding. Copyright protection represents the focus of most of these applications. An extensive description of the various applications is given in [1]. Over the last decade, watermarking research has focused mainly on developing news paradigms for watermark embedding and detection. However, in the last few years there was less obsession with this goal; rather, information-theoretic watermarking research began to emerge [2], [3]. In particular, a theory has recently been developed to establish the fundamental limits of the watermarking (data-hiding) problem. Around the same time, Cox et al. [6]

L. Ghouti is with the Department of Information and Computer Science, King Fahd University of Petroleum and Minerals, Dhahran 31261, Saud Arabia. Email: ghouti@ccse.kfupm.edu.sa

A. Bouridane is with the School of Computer Science, Queen's University of Belfast, BT7 1NN, UK. Email: A.Bouridane@qub.ac.uk

S. Boussakta is with School of Electronic and Electrical Engineering, University of Leeds, UK. Email: S.Boussakta@leeds.ac.uk have also recognized that one may view watermarking as communications with side information known at the encoder. This is reminiscent of the communications problem with a fixed noisy channel, and side information at the encoder [6]. Interestingly enough, Chen and Wornell [7] were the first to establish the analogy between watermarking and communications with side information problems. The goal of this paper is to derive the watermarking (data-hiding) capacity estimates for balanced multiwavelets using various statistical models for the host image. Section II outlines the mathematical properties of balanced multiwavelets and the motivation behind their use in digital watermarking applications. Section III describes the basic statistical model for the image watermarking problem. Then, we will derive the data-hiding capacity for balanced multiwavelets using various channel models in Section IV. Performance results of a spread spectrum watermarking system based on balanced multiwavelts are outlined in Section V. Section IV concludes this paper.

\section{Multiwavelets And Balanced Multiwavelets}

Orthogonality is a desirable property for software/hardware implementation and symmetry provides comfort to image perception [8]. In the context of image processing applications, the following three properties are important: 1) orthogonality to ensure the decorrelation of subband coefficients, 2) symmetry (i.e., linear phase) to process finite length signals without redundancy and artifacts, and 3) finite-length filters for computational efficiency. However, most real scalar wavelet transforms fail to possess these properties simultaneously. To circumvent these limitations, multiwavelets have been proposed where orthogonality and symmetry are allowed to co-exist by relaxing the time-invariance constraint [8].

\section{A. Multiwavelets}

Multiwavelets may be considered as generalization of scalar wavelets. However, some important differences exist between these two types of multiresolution transforms. In particular, whereas wavelets have a single scaling $\phi(t)$ and wavelet function $\psi(t)$, multiwavelets may have two or more scaling and wavelet functions. In general, $r$ scaling functions can be written using the vector notation $\Phi(t)=\left[\phi_{1}(t) \phi_{2}(t) \cdots \phi_{r}(t)\right]^{T}$, where $\Phi(t)$ is called the multiscaling function. In the same way, we can define the multiwavelet function using $r$ wavelet functions as $\Psi(t)=\left[\psi_{1}(t) \psi_{2}(t) \cdots \psi_{r}(t)\right]^{T}$. The scalar case is represented by $r=1$. Most of developed multiwavelet transforms use two scaling and wavelet functions, while $r$ can take theoretically any value. Similar to scalar wavelets and for 
the case where $r=2$, the multiscaling function satisfies the following two-scale equation:

$$
\begin{aligned}
& \Phi(t)=\sqrt{2} \sum_{k=-\infty}^{\infty} H_{k} \Phi(2 t-k), \\
& \Psi(t)=\sqrt{2} \sum_{k=-\infty}^{\infty} G_{k} \Phi(2 t-k),
\end{aligned}
$$

However, it should be noted that $\left\{H_{k}\right\}$ and $\left\{G_{k}\right\}$ are $2 \times 2$ matrix filters defined as:

$$
\begin{gathered}
H_{k}=\left[\begin{array}{ll}
h_{0}(2 k) & h_{0}(2 k+1) \\
h_{1}(2 k) & h_{1}(2 k+1)
\end{array}\right], \\
G_{k}=\left[\begin{array}{ll}
g_{0}(2 k) & g_{0}(2 k+1) \\
g_{1}(2 k) & g_{1}(2 k+1)
\end{array}\right]
\end{gathered}
$$

where $\left\{h_{k}(n)\right\}$ and $\left\{g_{k}(n)\right\}$ are the scaling and wavelet filter sequences such that $\sum_{n} h_{k}^{2}(n)=1$ and $\sum_{n} g_{k}^{2}(n)=1$ for $k=1,2$. The matrix elements in the filters, given by (3) and (4), provide more degrees of freedom than scalar wavelets. However, the multi-channel nature of multiwavelets yields a subband structure that is different from that using scalar wavelets.

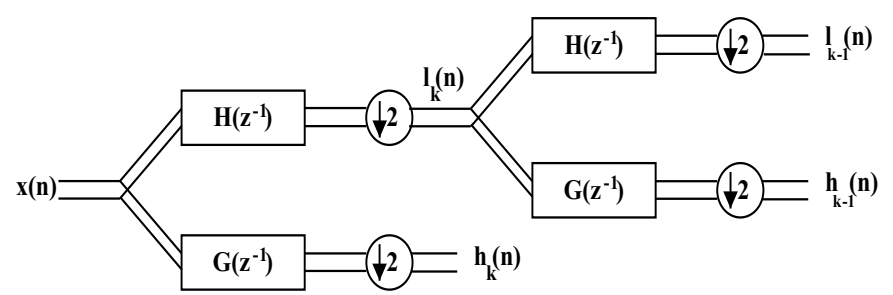

Fig. 1. Multiwavelet filter bank using one iteration.

Fig. 1 clearly shows that multiwavelets are defined for vector-valued signals (1D and $2 \mathrm{D}$ ). Such vectorizing does not only introduce a fundamental asymmetry but it yields an approximation subband that does not represent a coarse approximation of the input image. The structure of the latter is different from that obtained using scalar wavelets. In the case of multiwavelets, the four sub-blocks of the approximation subband have very dissimilar spectral characteristics [8].

\section{B. Balanced Multiwavelets}

Lebrun and Vetterli [8] indicate that the balancing order of the multiwavelet is indicative of its energy compaction efficiency. However, a high balancing order alone does not ensure good image compression performance. For a scalar wavelet, the number of vanishing moments of its wavelet function $\int t^{m} \psi(t) d t=0$ determines its vanishing order. For a scalar wavelet with vanishing order $K$, the highpass branch cancels a monomial of order less than $K$ and the lowpass branch preserves it. For a multiwavelet transform, we have the similar notion of approximation order; a multiwavelet is said to have an approximation order of $K$ if the vanishing moments of its wavelets, $\int t^{m} \psi_{i}(t) d t=0$ for $0 \leq i \leq r-1$ and $0 \leq m \leq K-1$. An approximation order of $K$ implies that the highpass branch cancels monomials of order less than $K$. But, in general, for multiwavelets, the preservation property does not automatically follow from the vanishing moments property. If the multifilter bank preserves the monomials at the lowpass branch output, the multiwavelet is said to be balanced [8]. The balancing order is $p$ if the lowpass and highpass branches in the filter bank preserve and cancel, respectively all monomials of order less than $p(p \leq K)$. Multiwavelets that do not satisfy the preservation/cancellation property are said to be unbalanced. For unbalanced multiwavelets, the input needs suitable prefiltering to compensate for the absence of the preservation/cancellation property, balancing obviates the need for input prefiltering; thus, they are computationally more efficient than the unbalanced multiwavelets. A time-varying representation of balanced multiwavelets is shown in Fig. 2.

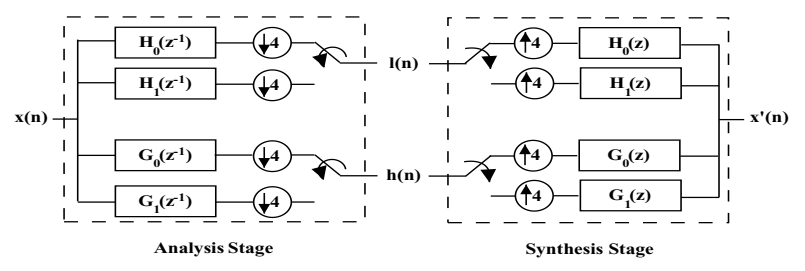

Fig. 2. Time-varying multiwavelet filter bank. Analysis stage (left). Synthesis stage (right).

The time-varying filter bank of Fig. 2 is defined by the following relations:

$$
\begin{aligned}
& {\left[\begin{array}{l}
H_{0}(z) \\
H_{1}(z)
\end{array}\right]=H\left(z^{2}\right)\left[\begin{array}{c}
1 \\
z^{-1}
\end{array}\right]} \\
& {\left[\begin{array}{l}
G_{0}(z) \\
G_{1}(z)
\end{array}\right]=G\left(z^{2}\right)\left[\begin{array}{c}
1 \\
z^{-1}
\end{array}\right]}
\end{aligned}
$$

where $H_{0}(z)$ and $H_{1}(z)$ are the Z-transforms of the two lowpass branch filters $h_{0}$ and $h_{1}$. Similarly, $G_{0}(z)$ and $G_{1}(z)$ are the Z-transforms of the two highpass branch filters $g_{0}$ and $g_{1}$. In the time-varying filter bank implementation, the coefficients of the two lowpass (highpass) filters are simply interleaved at the output (see Fig. 2). Therefore, a separable 2D transform can now be defined in the usual way as the tensor product of two 1D transforms [8]. It is worth noting that unlike their unbalanced counterparts, the sub-blocks of the approximation subband of balanced multiwavelets have similar spectral characteristics as shown in Fig. 3 for the case of Lena image.

\section{INFORMATION-THEORETIC DATA-HIDING ANALYSIS}

To derive the fundamental limits of watermarking and data hiding systems, we will follow the framework used in [2], [3], [4], [5] where no a priori assumptions are made about the embedding and decoding functions. The recent theory developed in [3], [5] establish the fundamental limits of the watermarking (and data hiding) problem. A communicationlike representation of the watermarking problem is shown in Fig. 4. 


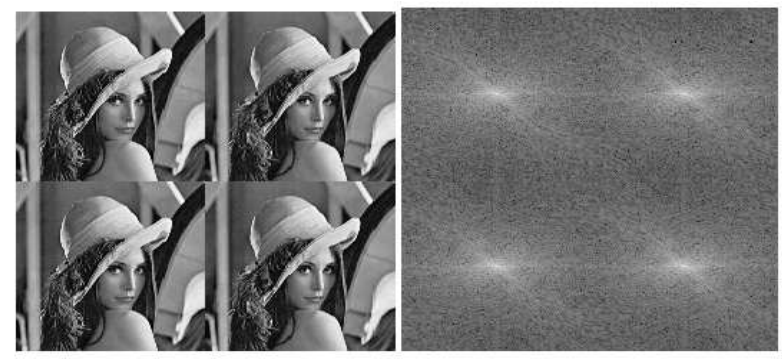

Fig. 3. Balanced multiwavelet approximation subband of Lena image (left) Spectral densities of subband blocks $L_{0} L_{0}, L_{0} L_{1}, L_{1} L_{0}$ and $L_{1} L_{1}$ (right)

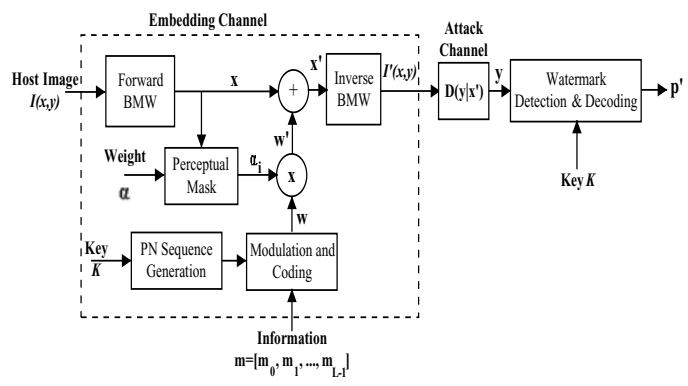

Fig. 4. Watermarking system model.

\section{A. Communications Model for Watermarking}

In Moulin-Mihçak's framework [3], [5], the watermarking system embeds or hides a watermark payload message $M$ in a length-N host data sequence $S^{N}=\left(S_{1}, \cdots, S_{N}\right)$. Sideinformation $K^{N}=\left(K_{1}, \cdots, K_{N}\right)$, such as cryptographic key or host signal-dependant data, may used by the watermark embedding stage. The watermarked data is denoted by $X^{N}=\left(X_{1}, \cdots, X_{N}\right)=f_{N}\left(S^{N}, M, K^{N}\right)$. Watermark attackers, modeled by attack channels, intend to remove or at least make useless the embedded message $M$. The sequence $Y^{N}=\left(Y_{1}, \cdots, Y_{N}\right)$ represents the attacked watermarked sequence. To derive the data-hiding capacity, we assume that the host images can be "correctly" modeled as sequences of independent and identically distributed (i.i.d.) $K$-dimensional Gaussian random vectors $S \sim \mathcal{N}(0, R)$, where $R$ is a $K \times$ $K$ correlation matrix. In this work, the squared Euclidean distance, $d(x, y)=\|x-y\|^{2}$, for $x, y \in \Re^{K}$ is used as the main distortion metric. Data-hiding capacity estimates for the scalar case, $K=1$ (in this case $S \sim \mathcal{N}\left(0, \sigma^{2}\right)$ are presented in [3]. In this paper, we are mainly interested in the parallel representation of the outlined problem. Thus, the host data $S$ is represented by means of $K$ parallel Gaussian channels. In the latter case, the channel inputs are $K$ independent sources $S_{k}, 1 \leq k \leq K$. Each channel $S_{k}$ is modeled as a sequence of i.i.d. Gaussian random variables $\mathcal{N}\left(0, \sigma_{k}^{2}\right)$. Because the watermarking problem can be viewed as a gametheoretic problem between the data embedder and the attacker who is an intelligent opponent, game-theoretic analysis of the watermarking problem has been successfully formulated for both the scalar and vector cases [4], [5]. In this game-theoretic framework [5], maximum distortion levels are specified for both the watermark embedder $\left(D_{1}\right)$ and attacker $\left(D_{2}\right)$. The maximum distortion imposed on the watermark embedder is given by [5]:

$$
E d^{N}\left(S^{N}, X^{N}\right) \leq D_{1}
$$

Attacks on embedded watermarks, modeled by specific channel models, are subject to distortion $D_{2}$ [5]:

$$
E d^{N}\left(S^{N}, Y^{N}\right) \leq D_{2}, \quad N \geq 1
$$

For a specific length- $N$ data-hiding code, the data-hiding capacity $C\left(D_{1}, D_{2}\right)$ is defined as the supremum of all achievable rates $R$ for distortions $\left(D_{1}, D_{2}\right)$ [5].

\section{B. Models of Typical Images}

Unlike in the case of unbalanced multiwavelets, the structure of the subbands emanating from balanced multiwavelet decomposition have similar structure to that obtained using scalar wavelet decomposition (refer to Section II for details). This similarity in subband structure motivates us to investigate the suitability of well-established statistical models that were initially designed for scalar wavelets. In these models [9], [10], subbands' coefficients are modeled as Gaussian and generalized-Gaussian processes, respectively, with zero means and variances that depend on the coefficient location within each decomposition subband. In [9], it is assumed that the coefficients' variances belong to a finite set of values $\sigma_{k}^{2}, 1 \leq$ $k \leq K$. In our work, we adopt the technique proposed in [5] to estimate representative values of $\sigma_{k}^{2}, 1 \leq k \leq K$. Fig. 5 shows the resulting 256 parallel channels in Lena image.

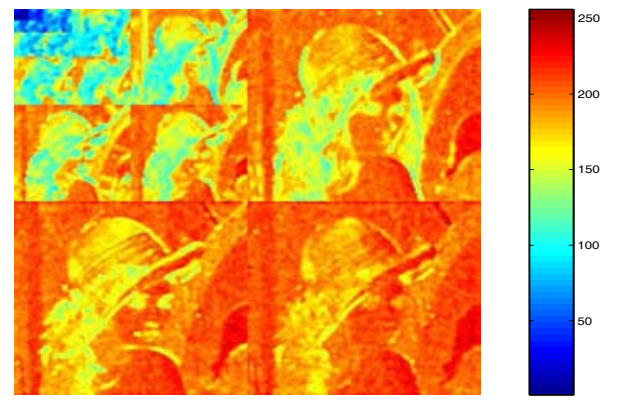

Fig. 5. EQ-estimated 256 parallel Gaussian channels in Lena image.

\section{Estimates of Data-Hiding Capacities}

In this section, we investigate the data-hiding capacity of typical natural test images. Analysis results are presented for four test images, Lena, Barbara, Baboon and Peppers. To derive the fundamental limits of watermarking and data hiding systems, we will follow the methodology used in [2], [3], [4], [5] where no a priori assumptions are made about the embedding and decoding functions. Capacity estimates for data-hiding are shown in Table I. Total data hiding capacity is denoted by $N C$. NC-Spike represents the same capacity using the spike model for the subband coefficients [11]. Table I clearly demonstrates that the BMW transforms yield higher data-hiding capacity due to the inherent structure of BMW transforms [8]. 


\begin{tabular}{|l|c||cc|cc|}
\hline \hline \multirow{2}{*}{ Image } & \multirow{2}{*}{$D_{1}$} & \multicolumn{2}{|c|}{$D_{2}=2 D_{1}$} & \multicolumn{2}{c|}{$D_{2}=5 D_{1}$} \\
\cline { 3 - 6 } & & $\mathrm{NC}$ & NC-Spike & NC & NC-Spike \\
\hline Lena (Daub-8) & 10 & 39911 & 31856 & 5305 & 6952 \\
Lena (9/7 filters) & & 43006 & 34215 & 5986 & 7377 \\
Lena (BMW BAT-1) & & $\mathbf{4 5 8 5 7}$ & $\mathbf{3 7 2 5 1}$ & $\mathbf{6 6 0 5}$ & $\mathbf{8 1 4 1}$ \\
\hline Baboon (Daub-8) & 25 & 81875 & 72773 & 15548 & 15587 \\
Baboon (9/7 filters) & & 85483 & 76998 & 17041 & 16507 \\
Baboon (BMW BAT-1) & & $\mathbf{8 6 3 9 3}$ & $\mathbf{7 7 6 6 4}$ & $\mathbf{1 7 1 5 0}$ & $\mathbf{1 6 4 8 5}$ \\
\hline Peppers (Daub-8) & 10 & 51912 & 35549 & 6341 & 6705 \\
Peppers (9/7 filters) & & 54963 & 38548 & 7117 & 7296 \\
Peppers (BMW BAT-1) & & $\mathbf{5 7 5 1 9}$ & $\mathbf{4 0 7 3 0}$ & $\mathbf{7 8 8 2}$ & $\mathbf{7 9 3 7}$ \\
\hline Barbara (Daub-8) & 20 & 32951 & 35339 & 5313 & 7899 \\
Barbara (9/7 filters) & & 33329 & 34686 & 5481 & 8018 \\
Barbara (BMW BAT-1) & & $\mathbf{4 3 1 5 2}$ & $\mathbf{4 4 9 1 7}$ & $\mathbf{7 1 6 8}$ & $\mathbf{9 7 9 9}$ \\
\hline \hline
\end{tabular}

TABLE I

TOTAL DATA-HIDING CAPACITIES (IN BITS) FOR IMAGES OF SIZE $N \times N=512 \times 512$ USING ORTHOGONAL DAUBECHIES $8,9 / 7$ LINEAR-PHASE FILTERS, AND BAT-1 BALANCED MULTIFILTERS.

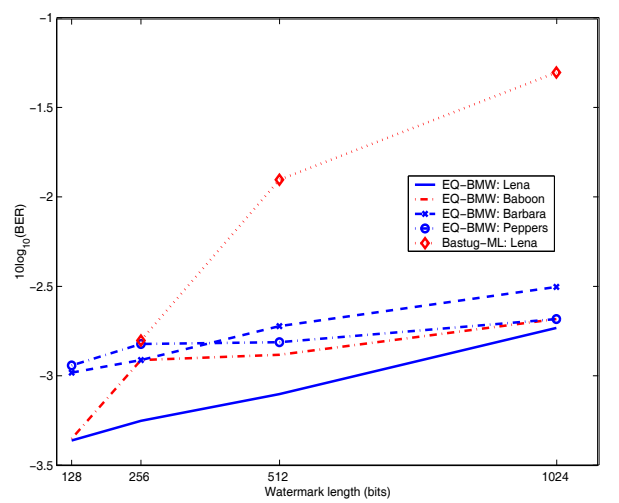

Fig. 6. Logarithmic BERs of repetition-coding using BMW method and block DCT for various watermark lengths ( $M=128,256,512$, and 1024).

\section{Simulation Results}

Using the data hiding estimates reported in this paper, we run experiments to evaluate the performance of a spread spectrum watermarking system based on BMW transforms. We test the performance of the watermarking system using various test images (Lena, Barbara, Peppers, and Baboon). Also, we provide comparison with another system based on block DCT model [12]. Fig. 6 shows the bit error rate (BER) of the spread spectrum watermarking system using the EQBMW model. It is clear that the proposed system outperforms that based on a block DCT model [12]. It is worth noting that while the block DCT model performs watermark decoding using an ML detector, the BMW-based system uses a simple correlator for watermark detection. In Fig. 7, we show results for the performance of the decoder in the presence of additive white Gaussian noise (AWGN) noise. The watermark message consists of 128 bits sequence.

\section{CONCLUSIONS}

In this paper, we have derived capacity estimates of datahiding for balanced multiwavelets. Unlike the case of image coding applications, the inherent structure of BMW decomposition can be used constructively to achieve higher data-hiding

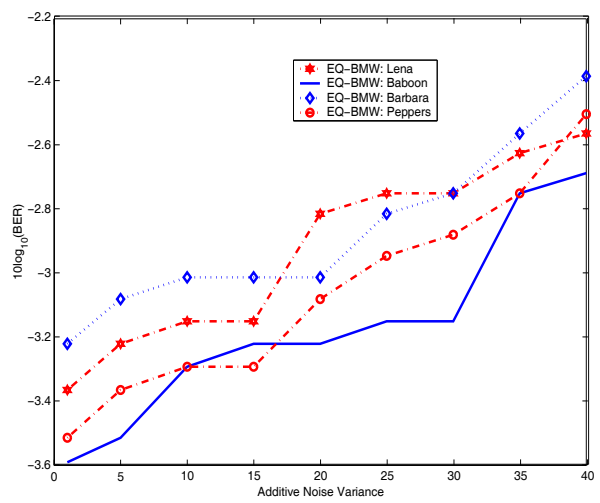

Fig. 7. Logarithmic BERs of encoded watermark code in the presence of AWGN noise using a watermark length of $128 \mathrm{bits}$.

capacities. Then, based on these estimates, we have evaluated the performance of a spread spectrum watermarking system based on BMW transforms. Performance results clearly how the inherent structure of BMW transform contribute to the improvement of watermark robustness.

\section{ACKNOWLEDGMENT}

The authors would like to thank Dr. Kivanç Mihçak for providing valuable help during the preparation of this paper. L. Ghouti acknowledges the support of KFUPM University.

\section{REFERENCES}

[1] I. J. Cox, M. L. Miller, and J. A. Bloom, "Watermarking applications and their properties," Presented at the International Conference on Information Technology, Las Vegas, 2000.

[2] P. Moulin and J. A. OSullivan, "Information-theoretic analysis of information hiding," IEEE Trans. Inform. Theory, vol. 49, no. 3, pp. 563-593, March 2003.

[3] P. Moulin and M. C. Mihçak, "A framework for evaluating the data-hiding capacity of image sources," IEEE Trans. Image Processing, vol. 11, no. 9, pp. 1029-1042, Sept. 2003

[4] A. S. Cohen and A. Lapidoth, "The Gaussian Watermarking Game," IEEE Trans. Inform. Theory, vol. 48, no. 6, pp. 1639-1667, June 2002.

[5] P. Moulin and M. C. Mihçak, "The Parallel-Gaussian Watermarking Game," IEEE Trans. Inform. Theory, vol. 50, no. 2, pp. 272-289, Feb. 2004.

[6] I. J. Cox, M. L. Miller, and A. L. McKellips, "Watermarking as communications with side information," Proc. IEEE, vol. 6, no.87, no. 7, pp. 1127-1141, July 1999.

[7] B. Chen and G. W. Wornell, "Quantization index modulation: A class of provably good methods for digital watermarking and information embedding," IEEE Trans. Inform. Theory, vol. 47, no. 5, pp. 1423-1443, May 2001.

[8] J. Lebrun and M. Vetterli, "Balanced multiwavelets: Theory and design," IEEE Trans. Signal Processing, vol. 46, no. 4, pp. 1119-1125, April 1998

[9] R. L. Joshi, H. Jafarkhani, J. H. Kasner, T. R. Fischer, N. Farvardin, M. W. Marcellin, and R. H. Bamberger, "Comparison of different methods of classification in subband coding of images," IEEE Trans. Image Processing, vol. 6, no. 11, pp. 1473-1486, Nov. 1997.

[10] S. LoPresto, K. Ramchandran, and M. T. Orchard, "Image coding based on mixture modeling of wavelet coefficients and a fast estimationquantization framework," in Proc. Data Compression Conf., 97, Snowbird, UT, pp. 221-230, 1997.

[11] C. Weidmann and M. Vetterli, "Rate-distortion analysis of spike processes," in Proc. Data Compression Conf., Snowbird, UT, March 1999.

[12] A. Baștuğ and B. Sankur, "Improving the payload of watermarking channels via LDPC coding," IEEE Signal Processing Letters, vol. 11, no. 2, pp. 90-92, Feb. 2004. 